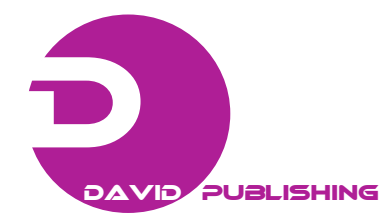

\title{
FMD Virus Genotyping Tool
}

\author{
Arafat Rahman*¹, Mohammad Arif Ashraf ${ }^{2,3}$, S.M. Sabbir Alam², ${ }^{1,}$, Farjana Khatun ${ }^{2,4}$, Saddam Hossain ${ }^{2}$, \\ Mosharraf Hossain ${ }^{2}$, Fokhruz Zaman², Munawar Sultana ${ }^{1}$ and M. Anwar Hossain ${ }^{1}$ \\ 1. Department of Microbiology, University of Dhaka, Dhaka, Bangladesh \\ 2. Bio-Bio-1 Bioinformatics Research Foundation, Dhaka, Bangladesh \\ 3. Deparment of Biochemistry and Molecular Biology, University of Dhaka, Dhaka, Bangladesh \\ 4. Department of Pharmacy, East West University, Dhaka, Bangladesh \\ *Corresponding author's e-mail: ac.arafat@gmail.com
}

\begin{abstract}
Foot and Mouth Disease Virus (FMDV) is an extremely contagious and rapidly evolving picornavirus infecting cloven-hoofed cattle, which was first recorded in Europe in 1544. FMDV endemic in Bangladesh causes annual loss approximately US\$125 million declining meat and milk productivity of cattle. FMDV has 7 distinct serotypes (A, O, C, Asia-1, SAT-1, SAT-2 and SAT-3) and about 61 sub-types. Immunity against one type does not confer protection against other types. It is essential to develop a novel vaccine against FMDV specific for circulating topotype in Bangladesh. So far 4 serotypes (A, O, C and Asia-1) and 1 sub-type (A22) have been identified in Bangladesh. A web-based genotyping tool can reduce the cost and time of wet-lab based serotyping experiments. FMDV genotyping tool uses a sliding window based local alignment scoring algorithm to detect genotype of a query sequence. This tool is developed in Python programming language using different standard library packages. Windowed subsequences of a query sequence are scored by BLAST2 program against a set of reference sequences. Three whole genome sequences from each 7 serotypes of FMDV are used to make the reference dataset. A graph is generated by plotting these scores against window index. Testing with FMDV genome sequences collected from NCBI shows that VP region of query sequence have highest score similarity with known serotypes of FMDV. VP region variability can be compared with reference sequences by the plot too. Genotype(s) of a region in query sequence can be inferred by looking at the best score from particular reference sequence represented by color bar in the output. This tool will also help to determine isolate's recombination pattern and quasi-species detection. FMDV web based genotyping tool can be used for quick identification of FMDV necessary to ensure food safety.
\end{abstract}

Key words: Foot and mouth disease, FMDV, genotype, serotype. 